\title{
Paroxysmal atrial fibrillation: changes in factor VIII and von Willebrand factor impose early hypercoagulability
}

Mariya Negrinova Negreva ${ }^{1}$, Krasimira Prodanova ${ }^{2}$, Katerina Vitlianova ${ }^{3}$, Christiana Madjova $^{4}$

${ }^{1}$ Department of Cardiology, Faculty of Medicine, Medical University "Prof. Dr. P. Stoyanov", Varna, Bulgaria

${ }^{2}$ Faculty of Applied Mathematics and Computer Science, Technical University of Sofia, Sofia, Bulgaria

${ }^{3}$ Clinic of Cardiology, Second City Hospital of Sofia, Sofia, Bulgaria

${ }^{4}$ Department of Conservative Dentistry and Oral Pathology, Faculty of Dental

Medicine, Medical University, Varna, Bulgaria

Submitted: 7 January 2020

Accepted: 23 May 2020

Arch Med Sci Atheroscler Dis 2020; 5: e140-e147

DOI: https://doi.org/10.5114/amsad.2020.97101

Copyright $\odot 2020$ Termedia \& Banach

\section{Abstract}

Introduction: Paroxysmal atrial fibrillation (PAF) is a well-documented prothrombotic state that carries significant embolic risk. However, precise hemostatic changes in the very early stage of the disease are not completely studied. The aim of the study was to study von Willebrand factor (vWF) and coagulation factor VIII (FVIII) plasma levels and activity in the first hours (up to $24 \mathrm{~h}$ ) of PAF clinical manifestation.

Material and methods: We selected consecutively 51 non-anticoagulated patients ( 26 men, 25 women, mean age: $59.84 \pm 1.60$ ) with PAF and 52 controls ( 26 men, 26 women, mean age: $59.50 \pm 1.46$ years) corresponding in gender, accompanying diseases and conducted treatment. The indicators were examined using enzyme-linked immunoassays and photometric tests. Results: All patients were hospitalized between the $2^{\text {nd }}$ and $24^{\text {th }} \mathrm{h}$ after the onset of arrhythmia (mean: $8.14 \pm 0.74 \mathrm{~h}$ ). Higher FVIII levels (107.52 $\pm 3.48 \%$ vs. $93.85 \pm 2.93 \%, p<0.05)$ and activity $(200.03 \pm 11.11 \%$ vs. $109.73 \pm 4.90 \%$, $p<0.001)$ were found in the PAF group. VWF levels $(178.40 \pm 12.95 \%$ vs. $119.53 \pm 6.12 \%, p<0.001)$ and activity $(200.92 \pm 12.45 \%$ vs. $110.80 \pm 5.14 \%$, $p<0.001$ ) were also higher. These changes did not depend on age, sex, body mass index or $\mathrm{CHA}_{2} \mathrm{DS}_{2}$-VASc score in the PAF group $(p>0.05)$. PAF duration was a significant predictor of increased FVIII levels and activity. Increased PAF duration was followed by increased values of the factors $(r=0.85, p<$ $0.001 ; r=0.83, p<0.001$.

Conclusions: The results presented an activated coagulation cascade and endothelial injury, suggesting hypercoagulability still in the early hours of PAF. These changes in PAF did not correlate with $\mathrm{CHA}_{2} \mathrm{DS}_{2}$-VASc score risk factors, outlining PAF as a possible independent embolic risk factor.

Key words: coagulation cascade, endothelial injury, paroxysmal atrial fibrillation.

\section{Introduction}

The hemostatic balance in the human body is the result of a precisely maintained equilibrium between the processes of fibrin formation and fibrinolysis [1]. Delayed blood flow, changes in vessel wall or blood composition can disturb this balance and lead to thrombotic complications. Despite their seemingly identical manifestation, namely the formation of

\author{
Corresponding author: \\ Assoc. Prof. Mariya Negrinova \\ Negreva \\ Department of Cardiology \\ Faculty of Medicine \\ Medical University \\ "Prof. Dr. P. Stoyanov" \\ Varna, Bulgaria \\ Phone: +359888478303 \\ E-mail: mnegreva@abv.bg
}


occlusive thrombi, the pathophysiological mechanisms that lead to these complications may be fundamentally different [2]. For example, the main cause of venous thromboembolism is delayed blood flow in the peripheral veins. The initiating moment in arterial thrombosis is platelet adhesion and aggregation due to endothelial damage. These fundamental differences are of major clinical importance because they predetermine a fundamentally different approach to antithrombotic therapy. Awareness of hemostasis in various prothrombotic conditions is the key to creating effective antiplatelet or anticoagulant therapies and, respectively, to successful prevention and treatment of thromboembolic events.

As a common pathophysiological mechanism of myocardial infarction, ischemic stroke, deep vein thrombosis, and pulmonary embolism, thrombosis is a leading cause of death [3]. As a well-defined prothrombotic condition, atrial fibrillation remains a major risk factor for two of these diseases: ischemic stroke and peripheral arterial thromboembolism. A systematic analysis of the Global Burden of Disease Study 2010 showed that the mortality associated with AF embologenic risk has increased two-fold in the last two years, reaching an age-adjusted mortality rate of 1.7 per 100,000 [4]. It is believed that one-quarter to two-thirds of the AF population are patients with transient or paroxysmal atrial fibrillation (PAF) [5]. This raises significant interest in PAF embolic risk and associated hemostatic changes.

Generally, PAF thromboembolic incidents with onset < $48 \mathrm{~h}$ have a low incidence rate $(0-0.9 \%)$; therefore acute cardioversion is justified for the general population [6-9]. However, the risk appears to vary substantially depending on the patient's characteristics and the onset of the arrhythmia even within those first $48 \mathrm{~h}$ of the disease [10]. It is unacceptably high in patients with heart failure and diabetes mellitus (up to $9.8 \%$ ) compared to patients $<60$ years of age without heart failure. After the $12^{\text {th }} \mathrm{h}$ from the onset of arrhythmia, the risk reaches $1.1 \%$ compared to $0.3 \%$ in the first few hours of the disease. Very little is clear about the hemostatic changes in the early hours of AF and their relationship with the onset of the disease.

Coagulation factor VIII (FVIII) and von Willebrand factor (VWF) are important molecules for primary and secondary hemostasis. It is well known that congenital and acquired diseases associated with their deficiency, lack or reduced activity, are associated with significant, sometimes life-threatening bleeding [11]. They play an important role in the thrombus formation process and their hemostatic functions are closely related and interdependent. FVIII is a key factor in the coagulation cascade and therefore its activity and concentration are determi- nants of hemostatic balance [12]. Most frequently activated by thrombin, FVIIla has the function of a non-enzymatic FIX co-factor, causing significant activation and a burst of thrombin formation, the molecule that directly stimulates fibrin synthesis. The von Willebrand factor is directly responsible for the FVIII procoagulant function. By binding it into the non-covalent FVIII/vWF complex, it stabilizes it and significantly prolongs its circulation half-life. vWF plasma levels are associated with the presence of endothelial damage, since endothelial cells are their primary source [13].

The pivotal role of FVIII and VWF in hemostasis and the lack of sufficient knowledge of it in the early hours of PAF were prerequisites for our study.

The aim of the study was to study VWF and FVIII plasma levels and activity in the first hours (up to $24 \mathrm{~h}$ ) of the clinical manifestation of PAF.

\section{Material and methods}

\section{Study population}

Patients over 18 years of age with PAF episode onset of $<24 \mathrm{~h}$ prior to hospitalization were sequentially screened. They were diagnosed based on electrocardiographic study performed at the hospital. The study was conducted in the Intensive Cardiology Unit of the First Cardiology Clinic at the University Hospital St. Marina, Varna, for the period 10.2010-05.2012 after approval by the local ethics committee (9/14.10.2010) and in accordance with the Declaration of Helsinki [14]. Based on the inclusion and exclusion criteria presented below, 51 patients (26 men and 25 women) with a mean age of $59.84 \pm 1.60$ years (31-77 years) were sequentially selected.

A control group of volunteers with no anamnestic or electrocardiographic AF data was formed, applying the same exclusion criteria (see below). Of the 169 screened volunteers, 52 were selected for the study. Their mean age was $59.50 \pm 1.46$ years (30-76 years), 26 men and 26 women.

The selection of participants was aimed at balancing as much as possible between the two groups the factors influencing the studied indicators and thus eliminating their effect on the results obtained. The two groups were created to be identical in terms of sex, age, body mass index (BMI), deleterious habits, comorbidities and treatment. These are indicators which may affect the values of the studied indicators.

Each participant was enrolled in the study after signing informed consent.

\section{Inclusion criteria}

1. Ability to clearly define the onset of arrhythmia, continuing at the time of hospitalization.

2. Lack of exclusion criteria. 


\section{Exclusion criteria}

1. Cardiovascular diseases: ischemic heart disease, heart failure, high-grade and /or uncontrolled hypertension, moderate or severe acquired valve defects, cardiomyopathy, implanted device for the treatment of rhythm-conduction disorders, inflammatory heart disease, congenital heart diseases.

2. Other diseases - kidney or liver failure, inflammatory and/or infectious diseases, neoplastic and autoimmune diseases, chronic pulmonary insufficiency, endocrine disorders (except for non-insulin dependent, well-controlled DM type 2); previous thromboembolic incidents, bleeding diathesis, miscarriages (for women).

3. Intake of hormone replacement therapy, contraceptives, oral anticoagulants or antiplatelet drugs, pregnancy, systemic intake of analgesics (incl. NSAIDs), obesity with BMI > $35 \mathrm{~kg} / \mathrm{m}^{2}$.

4. Unsuccessful restoration of sinus rhythm with drugs (propafenone) (for the patient group).

\section{Blood sampling and laboratory measurements}

Peripheral venous blood was collected once from each participant in the study in $3.2 \%$ sodium citrate Vacutainers (VACUETTE, Greiner Bio-One North America, Inc.). In patients the blood sample was taken immediately after hospitalization and in controls during the outpatient examination. After centrifugation of the samples at $2500 \mathrm{~g}$ for $15 \mathrm{~min}$, the plasma was pipetted and stored at $-20^{\circ} \mathrm{C}$ for a maximum of 1 month.

Each indicator was determined twice and the average was taken into account when calculating the results.

Quantification of FVIII was made using an enzyme-linked immunosorbent assay (ASSERACHROM VIII: Ag, Diagnostica Stago, Asnières sur Seine, France). Factor activity was determined photometrically (TECHNOCHROM FVIII, Technoclone, Vienna, Austria). Levels of VWF antigen (vWF : Ag) were examined by TECHNOZYM vWF: Ag ELISA, Technoclone, Vienna, Austria. Activity of the indicator was measured on the basis of its capacity to bind collagen, a major physiological function of VWF that determines its adhesive properties. For this purpose we used an ELISA test (TECHNOZYM vWF : CBA, ELISA, Vienna, Austria).

The intra-assay coefficient of variation (CV) was $<5 \%$, except for vWF : Ag (5.5\%). The inter-assay CV was $<5 \%$, except for VWF : Ag (5.9\%). The lower limit of detection was $0.5 \%$ for FVIII : Ag, 0\% for FVIII activity, $0.01 \%$ for VWF : Ag and $0.01 \%$ for VWF : act.

\section{Statistical analysis}

All analyses were conducted using Statistica 13.3.0, StatSoft Inc, USA.

Continuous variables were expressed as mean \pm standard error of the mean (SE) and categorical variables were expressed as percentage of the total group. Normality of distribution was assessed by the Kolmogorov-Smirnov (Lilliefors) test. Two-tailed Student's $t$-test for independent samples was used to compare quantitative variables. Fisher's exact or Pearson's $\chi^{2}$ test was used to compare categorical variables and occurrence frequency. Values of $p<$ 0.05 were considered statistically significant. Linear regression analysis was used to identify dependencies in PAF between FVIII and VWF plasma levels and activity (dependent continuous variables) and the explanatory continuous variables age, BMI and onset of the arrhythmia (measured as time spent in AF until hospitalization). The relationship between hemostatic indicators and the categorical independent variables sex and $\mathrm{CHA}_{2} \mathrm{DS}_{2}$-VASc score was examined using analysis of variance (ANOVA). Variables showing a level of association $p<0.05$ were considered as prognostic.

\section{Results}

\section{Study population}

There was no statistically significant difference between the patient and control groups in terms of sex, age, comorbidities and treatment, deleterious habits, BMI and main laboratory markers $(p>0.05)$ (Table I) [15]. The transthoracic echocardiography indicators also showed no significant differences ( $p>0.05$ ) (Table II).

PAF-episode onset analysis showed that patients were hospitalized between the $2^{\text {nd }}$ and the $24^{\text {th }} \mathrm{h}$ from the onset of the arrhythmia, most often at the $5^{\text {th }} \mathrm{h}$. The mean duration of episodes until hospitalization was $8.14 \pm 0.76 \mathrm{~h}$.

\section{Plasma levels and activity of vWF and FVIII}

Higher plasma levels of FVIII (107.52 $\pm 3.48 \%$ vs. $93.85 \pm 2.93 \%, p<0.05$; Figure 1$)$ and higher activity $(200.03 \pm 11.11 \%$ vs. $109.73 \pm 4.90 \%$, $p<0.001$; Figure 2) were detected in PAF compared to controls in sinus rhythm. The calculated level of the power was 0.80 (often considered the minimum acceptable level) and 0.99 for FVIII levels and activity, respectively.

VWF levels were significantly higher in the patient group compared to the control group (178.40 $\pm 12.95 \%$ vs. $119.53 \pm 6.12 \%, p<0.001$; Figure 3 ). VWF : CBA was also higher in the PAF group (200.92 $\pm 12.45 \%$ vs. $110.80 \pm 5.14 \%, p<0.001$; Figure 4). The levels of the power of tests were 0.89 and 0.99 , respectively. 
Table I. Clinical characteristics of participants

\begin{tabular}{|c|c|c|c|}
\hline Parameter & Patients with PAF & Control group & $P$-values \\
\hline Number of participants & 51 & 52 & 0.89 \\
\hline Mean age [years] & $59.84 \pm 1.60$ & $59.50 \pm 1.46$ & 0.87 \\
\hline Men/women & $26 / 25$ & $26 / 26$ & 0.93 \\
\hline Hemoglobin $[\mathrm{g} / \mathrm{l}]$ & $140.20 \pm 1.78$ & $136.17 \pm 1.90$ & 0.13 \\
\hline Leukocyte count $\left[\times 10^{9} / 1\right]$ & $8.01 \pm 0.23$ & $7.30 \pm 0.31$ & 0.07 \\
\hline Platelet count $\left[\times 10^{9} / \mathrm{l}\right]$ & $244.70 \pm 8.41$ & $228.42 \pm 7.24$ & 0.14 \\
\hline $\mathrm{eGFR}\left[\mathrm{ml} / \mathrm{min} / 1.73 \mathrm{~m}^{2}\right]$ & $78.98 \pm 2.72$ & $83.17 \pm 1.62$ & 0.19 \\
\hline Systolic blood pressure [mm Hg] & $132.30 \pm 2.96$ & $125.25 \pm 2.08$ & 0.05 \\
\hline Diastolic blood pressure [mm Hg] & $78.43 \pm 1.14$ & $76.77 \pm 1.04$ & 0.09 \\
\hline \multicolumn{4}{|l|}{ Accompanying diseases: } \\
\hline Hypertension & $37(72.54 \%)$ & $34(65.38 \%)$ & 0.44 \\
\hline Diabetes mellitus type 2 & $3(5.88 \%)$ & $2(3.84 \%)$ & 0.62 \\
\hline Dyslipidemia & $4(7.84 \%)$ & $3(5.77 \%)$ & 0.69 \\
\hline \multicolumn{4}{|c|}{ Medicaments for hypertension and dyslipidemia: } \\
\hline Beta blockers & $19(37.25 \%)$ & $17(32.69 \%)$ & 0.62 \\
\hline ACE inhibitors & $15(29.41 \%)$ & $14(26.92 \%)$ & 0.78 \\
\hline Sartans & $11(21.57 \%)$ & $9(17.31 \%)$ & 0.58 \\
\hline Statins & $4(7.84 \%)$ & $3(5.77 \%)$ & 0.69 \\
\hline \multicolumn{4}{|l|}{ Deleterious habits: } \\
\hline Smoking & $8(15.69 \%)$ & $7(13.46 \%)$ & 0.75 \\
\hline Alcohol intake & $7(13.72 \%)$ & $6(11.53 \%)$ & 0.74 \\
\hline $\mathrm{BMI}\left[\mathrm{kg} / \mathrm{m}^{2}\right]$ & $23.85 \pm 0.46$ & $24.95 \pm 0.45$ & 0.09 \\
\hline $\mathrm{CHA}_{2} \mathrm{DS}_{2}$-VASc score ${ }^{*}$ & & No score & \\
\hline Number of patients with score $<2$ & 25 & & \\
\hline Number of patients with score $\geq 2$ & 26 & & \\
\hline
\end{tabular}

Table II. Echocardiographic parameters of participants

\begin{tabular}{|lccc|}
\hline Echocardiographic indicators & Patients with PAF & Control group & $P$-values \\
\hline LVEDD [mm] & $52.57 \pm 0.58$ & $52.29 \pm 0.57$ & 0.73 \\
\hline LVESD [mm] & $34.43 \pm 0.56$ & $34.73 \pm 0.48$ & 0.69 \\
\hline EF (\%) & $62.98 \pm 0.70$ & $61.54 \pm 0.58$ & 0.12 \\
\hline IVS [mm] & $10.37 \pm 0.23$ & $9.92 \pm 0.26$ & 0.20 \\
\hline PW [mm] & $10.24 \pm 0.21$ & $9.73 \pm 0.28$ & 0.16 \\
\hline LA volume [m//m²] & $22.81 \pm 0.45$ & $23.82 \pm 0.48$ & 0.13 \\
\hline RVEDD [mm] & $30.54 \pm 1.58$ & $29.17 \pm 1.52$ & 0.18 \\
\hline
\end{tabular}


Scatterplot of FVIII levels - controls against FVIII levels - patients FVIII levels - controls $=81.8665+0.1088 x$

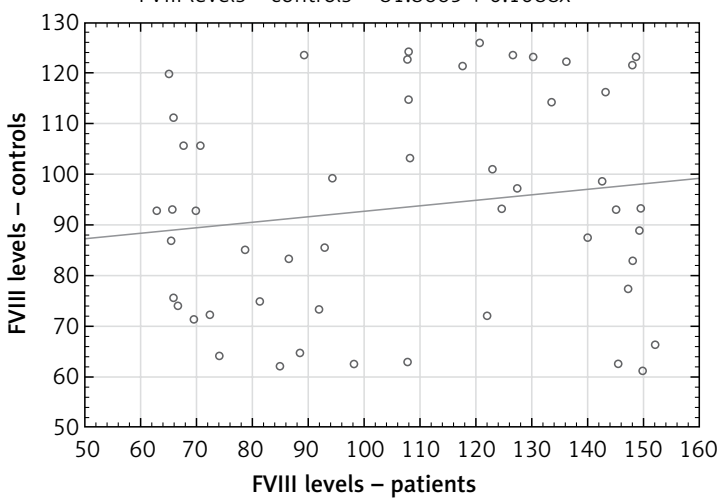

Figure 1. Scatterplot of FVIII plasma levels in PAF patients and controls

Each scatterplot presents a "round cloud" of points, not a systemic shape (e.g., a straight line, a curve etc.), the variables not being related.

Linear regression analysis showed that patients' age and BMI were not predictive variables for plasma FVIII levels $(r=0.07, p>0.05 ; r=0.06$, $p>0.05$, respectively), plasma FVIII activity $(r=$ $0.04, p>0.05 ; r=0.09, p>0.05$, respectively), plasma vWF levels $(r=0.13, p>0.05 ; r=0.07$, $p>0.05$, respectively), and VWF plasma activity ( $r=0.03, p>0.05 ; r=0.02, p>0.05$, respectively). The time from the onset of the arrhythmia was a significant predictor of increased FVIII and VWF plasma levels and activity, and increased PAF duration was followed by increased values of the factors $(r=0.85, p<0.001$, Figure $5 ; r=0.83$, $p<0.001$; Figure 6). No linear relationship was found between vWF levels and activity and time from the arrhythmia onset $(r=0.14, p>0.05 ; r=$ $0.12, p>0.05$ ).

ANOVA showed that there were no significant differences between male and female FVIII plasma levels and activity in the PAF group (107.72 $\pm 6.92 \%$ vs. $107.23 \pm 5.50 \%, p>0.05 ; 197.28 \pm 18.19 \%$ vs.

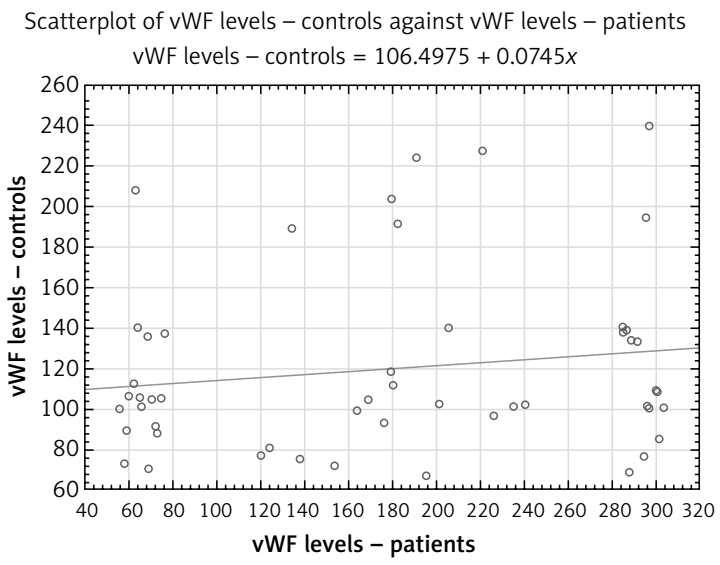

Figure 3. Scatterplot of VWF plasma levels in PAF patients and controls

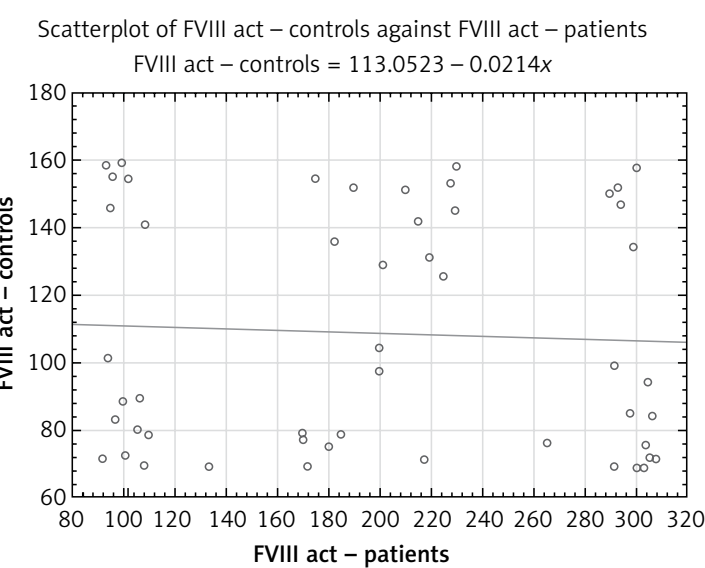

Figure 2. Scatterplot of FVIII coagulation activity in PAF patients and controls (FVIII act - FVIII activity)

$202.70 \pm 13.35 \%, p>0.05$; respectively), as well as vWF plasma levels and activity (169.07 $\pm 19.43 \%$ vs. $187.30 \pm 17.41 \%, p>0.05 ; 195.55 \pm 17.25 \%$ vs. $206.11 \pm 18.30, p>0.05$; respectively). No statistically significant difference was found between patients with low $\left(\mathrm{CHA}_{2} \mathrm{DS}_{2}-\mathrm{VASc}\right.$ score $\left.<2\right)$ and high embologenic risk ( $\mathrm{CHA}_{2} \mathrm{DS}_{2}$-VASc score $\left.\geq 2\right)$ in terms of FVIII plasma levels and activity (106.64 $\pm 5.70 \%$ vs. $108.27 \pm 6.67 \%, p>0.05 ; 202.02$ $\pm 13.88 \%$ vs. $198.14 \pm 17.50 \%, p>0.05$; respectively) and VWF plasma levels and activity $(191.79 \pm 17.51 \%$ vs. $165.45 \pm 19.02 \%, p>0.05 ; 202.02 \pm 18.56 \%$ vs. $199.89 \pm 17.14 \%, p>0.05$; respectively).

\section{Discussion}

Factor VIII is a coagulation factor whose procoagulant effect is well recognized. Its role in prothrombotic syndromes, such as myocardial infarction, deep vein thrombosis (DVT) and acute ischemic stroke, has been demonstrated [16]. Although it circulates in the blood in its inactive "sleeping" form bound in the FVIII/vWF complex, its plasma concentrations directly correlate with the manifestation of a number of thrombotic events.

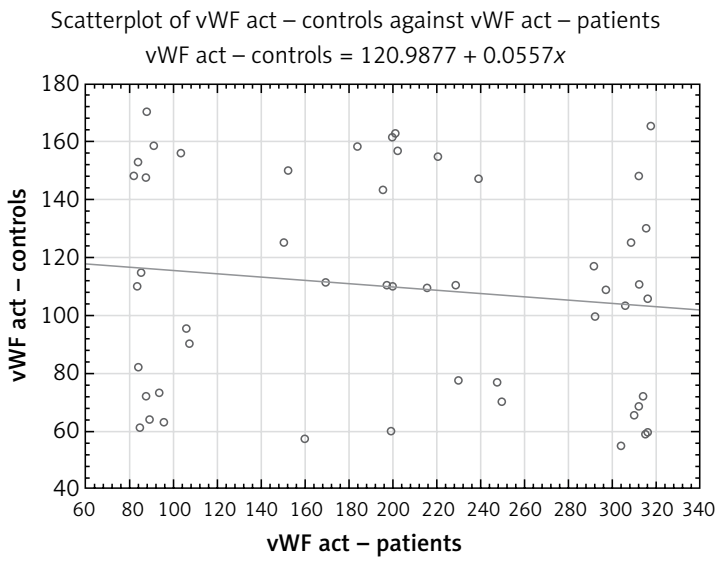

Figure 4. Scatterplot of VWF coagulation activity in PAF patients and controls (VWF act - VWF activity measured as collagen-binding activity) 
FVIII levels - patients vs. time - patients FVIII levels - patients $=67.458+4.9056 \times$ time - patients Correlation: $r=0.85108$

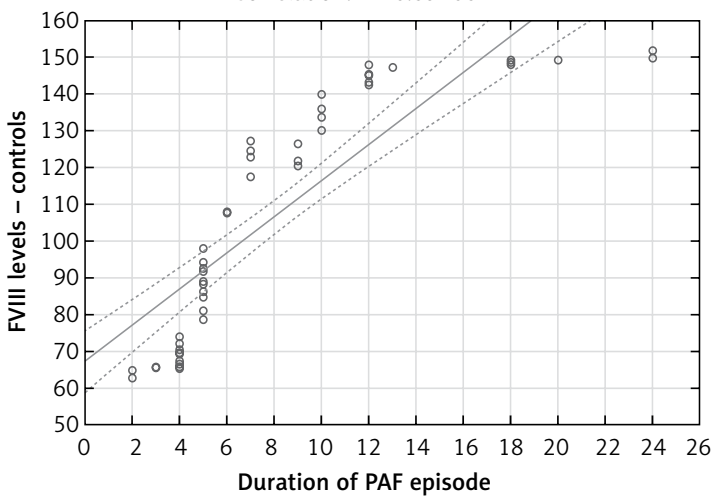

Figure 5. Dependence of FVIII levels on the duration of PAF episode

A strong relationship was found between elevated FVIII levels and the incidence of "de novo" or recurrent non-malignant DVT [17]. Every $10 \mathrm{lU} / \mathrm{dl}$ above normal limits increased the risk of the disease by $8 \%$. High plasma FVIII activity is commonly observed in patients with cerebral venous thrombosis and is considered a risk factor for this disease [18]. FVIII is associated not only with venous but also with arterial thrombotic complications. It defines an independently significant risk of acute coronary thrombosis in the absence of underlying coronary disease [19]. It is well known that its plasma levels are also associated with the risk of acute ischemic stroke and its consequences [20].

A prerequisite for the scientific interest in the FVIII molecule in the clinical manifestation of AF is the high risk of stroke and thromboembolism that the disease determines. It is well known that FVIII significantly activates the coagulation cascade in response to external and internal procoagulant stimuli [21]. Both thrombin and FXa within the outer TF-FVIIa-FXa initiation complex activate FVIII, and this largely depends on the concentration of the factor itself [22]. In this sense, the functional adequacy of FVIII depends not only on the activity but also on the levels of the molecule. Studies on the coagulation cascade in AF show significantly elevated levels of FVIII [23, 24]. Some of them also establish thromboembolic complications in the presence of high FVIII [25]. The studied populations were heterogeneous in terms of arrhythmia onset (patients with paroxysmal and persistent atrial fibrillation, as well as with restored sinus rhythm) and accompanying diseases. There are no studies on FVIII in PAF. There are no studies in the entire AF population that simultaneously evaluate procoagulant activity and coagulation factor levels. Although there is usually a direct cause-and-effect relationship between them, each of these indicators has its own value and provides specific clinical information [26].

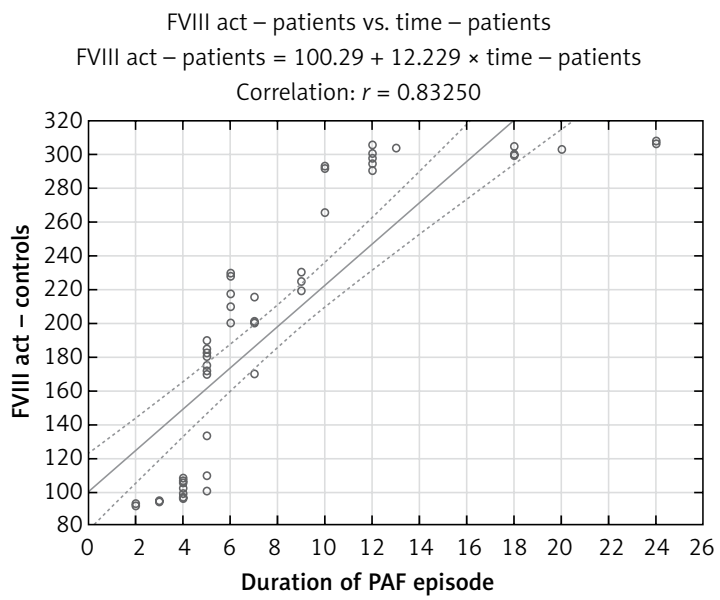

Figure 6. Dependence of FVIII activity on the duration of PAF episode (FVIII act - VIII)

This was the reason to simultaneously examine them in our study. The results showed that there were significant deviations in FVIII during the first $24 \mathrm{~h}$ of PAF (Figures 1 and 2). The coagulation activity of the factor was higher compared to the control group (Figure 2). Its plasma levels were also elevated (Figure 1). The unidirectional changes in both indicators and the known causal relationship between them give reason to believe that increased synthesis and secretion of FVIII were observed in PAF under conditions of procoagulant stimulation.

Although not directly involved in the enzyme reactions, but acting as a non-enzymatic co-factor, FVIII is a key coagulation cascade molecule. It is the main molecule responsible for the process of platelet propagation, regardless of the pathway in which the coagulation cascade is activated [16]. In this sense, the observed higher FVIII activity in the patient group implies significant activation of the coagulation cascade and a tendency for hypercoagulability in the first $24 \mathrm{~h}$ of the disease. The results of the regression analysis are extremely interesting. They clearly indicate that the clinical manifestation of PAF is associated with increased FVIII activity and levels regardless of age, sex, $B M I$, and embologenic risk, as determined by the $\mathrm{CHA}_{2} \mathrm{DS}_{2}$-VASc score $(p>0.05)$. PAF itself determines hypercoagulability, increasing over time (Figures 5, 6). Specific to FVIII structure is the volatility of its free form, which greatly limits and reduces its effective participation in the coagulation process. The binding of FVIII to $\mathrm{VWF}$ at a $1: 1$ ratio in the non-covalent FVIII/vWF complex prevents early proteolytic degradation of the coagulation factor and prolongs its circulation half-life. The VWF molecule is essential for the functionality of FVIII [27]. This was the reason for the simultaneous study of both VWF and FVIII in our study. High plasma vWF levels were detected (Figure 3). They 
are consistent and contribute to the unidirectional deviations in FVIII levels. It is reasonable to assume that high FVIII concentration is associated with prolonged plasma half-life and stabilization of the molecule.

VWF is released in the plasma mainly by endothelial cells and, to a lesser extent, by activated platelets in endothelial damage [28]. Due to Gplb conformational changes upon contact with subendental collagen, vWF is activated and has the ability to adhere to platelets. Increased vWF levels in the study patient group are indicative of endothelial damage in the first $24 \mathrm{~h}$ of PAF (Figure 3), and its increased activity (Figure 4) of enhanced platelet adhesion.

Changes in VWF, as well as in the aforementioned FVIII, are independent of sex, age, BMI and embologenic $\mathrm{CHA}_{2} \mathrm{DS}_{2}$-VASc score $(p>0.05)$. There was no correlation with the duration of arrhythmia $(p>0.05)$.

These results suggest a link between these factors and local endothelial burden, observed in high frequency uncoordinated atrial contraction and resulting from AF itself [29]. Changes in $\checkmark W F$ are part of the procoagulant state, formed in the early hours of PAF, which is independent of the well-known predictors of sex, age, BMI and $\mathrm{CHA}_{2} \mathrm{DS}_{2}$-VASc score.

The aim of our study was to investigate FVIII and VWF plasma activity and levels in the early hours of the clinical manifestation of PAF, namely the first $24 \mathrm{~h}$ from the onset of the disease. Therefore, we studied only patients who clearly specified that the arrhythmia started $<24 \mathrm{~h}$ prior to hospitalization. In this sense the presented results provide information exclusively about the coagulation in the early hours of clinical manifestation of PAF. They cannot in any way show or predict the coagulation activity in the later hours of the disease. We also believe that these limitations are a ground for new studies, which could examine the dynamics of the system.

In conclusion, the results presented an activated coagulation cascade and endothelial injury, suggesting hypercoagulability still in the early hours of PAF. These changes in PAF did not correlate with $\mathrm{CHA}_{2} \mathrm{DS}_{2}$-VASc score risk factors, indicating PAF as a possible independent embolic risk factor.

\section{Conflict of interest}

The authors declare no conflict of interest.

\section{References}

1. Dorgalaleh A, Daneshi M, Rashidpanah J, Roshani Yasaghi E. An overview of hemostasis. In: Congenital Bleeding Disorders. Dorgalaleh A (ed.). Springer International Publishing, Cham 2018; 3-26.
2. Koupenova M, Kehrel BE, Corkrey HA, Freedman JE. Thrombosis and platelets: an update. Eur Heart J 2017; 38: 785-91.

3. ISTH Steering Committee for World Thrombosis Day. Thrombosis: a major contributor to global disease burden. Thromb Res 2014; 134: 931-8.

4. Lozano R, Naghavi M, Foreman K, et al. Global and regional mortality from 235 causes of death for 20 age groups in 1990 and 2010: a systematic analysis for the Global Burden of Disease Study 2010. Lancet 2012; 380: 2095-128.

5. Chiang CE, Naditch-Brûlé L, Murin J, et al. Distribution and risk profile of paroxysmal, persistent, and permanent atrial fibrillation in routine clinical practice: insight from the real-life global survey evaluating patients with atrial fibrillation international registry. Circ Arrhythm Electrophysiol 2012; 5: 632-9.

6. Burton JH, Vinson DR, Drummond K, Strout TD, Thode HC, McInturff JJ. Electrical cardioversion of emergency department patients with atrial fibrillation. Ann Emerg Med 2004; 44: 20-30.

7. Crijns HJGM, Weijs B, Fairley AM, et al. Contemporary real life cardioversion of atrial fibrillation: results from the multinational RHYTHM-AF study. Int J Cardiol 2014; 172: 588-94.

8. Michael JA, Stiell IG, Agarwal S, Mandavia DP. Cardioversion of paroxysmal atrial fibrillation in the emergency department. Ann Emerg Med 1999; 33: 379-87.

9. Airaksinen KEJ, Grönberg T, Nuotio I, et al. Thromboembolic complications after cardioversion of acute atrial fibrillation: the FinCV (Finnish CardioVersion) study. J Am Coll Cardiol 2013; 62: 1187-92.

10. Nuotio I, Hartikainen JEK, Grönberg T, Biancari F, Airaksinen KE. Time to cardioversion for acute atrial fibrillation and thromboembolic complications. JAMA 2014; 312: 647-9.

11. Goodeve AC, Rosén S, Verbruggen B. Haemophilia A and von Willebrand's disease. Haemophilia 2010; 16 Suppl 5: 79-84.

12. Christopher N, Motto DG, Di Paola J. Diagnostic approach to von Willebrand disease. Blood 2015; 125: 2029-37.

13. Giblin JP, Hewlett LJ, Hannah MJ. Basal secretion of von Willebrand factor from human endothelial cells. Blood 2008; 112: 957-64.

14. World Medical Association Declaration of Helsinki. Ethical principles for medical research involving human subjects. 59th WMA General Assembly, Seoul 2008.

15. Kirchhof P, Benussi S, Kotecha D, et al.; ESC Scientific Document Group. 2016 ESC Guidelines for the management of atrial fibrillation developed in collaboration with EACTS. Eur J Cardiothorac Surg 2016; 50: e1-88.

16. Siegler JE, Samai A, Albright K, Boehme AK, MartinSchild S. Factoring in factor VIII with acute ischemic stroke. Clin Appl Thromb Hemost 2015; 21: 597-602.

17. Kyrle PA, Minar E, Hirschl M, et al. High plasma levels of factor VIII and the risk of recurrent venous thromboembolism. N Engl J Med 2000; 343: 457-62.

18. Vecht L, Zuurbier SM, Meijers JCM, Coutinho JM. Elevated factor VIII increases the risk of cerebral venous thrombosis: a case-control study. J Neurol 2018; 265: 1612-7.

19. Bosma J, Rijbroek A, Rauwerda JA. A rare case of thromboembolism in a 21-year old female with elevated factor VIII. Eur J Endovasc Surg 2007; 34: 592-4.

20. Kuo CY, Lin CH, Kuo YW, et al. Factor VIII levels are associated with ischemic stroke, stroke subtypes and neurological worsening. Curr Neurovasc Res 2015; 12: 85-90. 
21. Kamikubo Y, Mendolicchio GL, Zampolli A, et al. Selective factor VIII activation by the tissue factor-factor VIla-factor Xa complex. Blood 2017; 130: 1661-70.

22. Wang W, Wang YJ, Kelner DN. Coagulation factor VIII: structure and stability. Int J Pharm 2003; 259: 1-15.

23. Tóth NK, Csanádi Z, Hajas O, et al. Intracardiac hemostasis and fibrinolysis parameters in patients with atrial fibrillation. BioMed Res Int 2017; 2017: 1-10.

24. Wu N, Tong S, Xiang Y, et al. Association of hemostatic markers with atrial fibrillation: a meta-analysis and meta-regression. PLoS One 2015; 10: e0124716.

25. Hammwöhner M, Tautenhahn J, Kuester D, Goette A. Inadvertend catch of a factor VIII rich thrombus with a femoral sheath during cardiac catheterization. Int J Cardiol 2008; 130: e39-41.

26. O'Donnell J, Tuddenham EG, Manning R, KemballCook G, Johnson D, Laffan M. High prevalence of elevated factor VIII levels in patients referred for thrombophilia screening: role of increased synthesis and relationship to the acute phase reaction. Thromb Haemost 1997; 77: 825-8.

27. Peyvandi F, Garagiola I, Baronciani L. Role of von Willebrand factor in the haemostasis. Blood Transfus 2011; 9 (Suppl 2): s3-8.

28. Yee A, Kretz CA. Von Willebrand factor: form for function. Semin Thromb Haemost 2014; 40: 17-27.

29. Guazzi M, Arena R. Endothelial dysfunction and pathophysiological correlates in atrial fibrillation. Heart 2009, 95: 102-6. 